26

liberi, da una parte uu tubo col gaz idrogene, dall' altra un tubo con gaz ossigene. E' congiungendo le due lamine estreme di questi tubi che Grôve ha ottenuto da una pila di 50 elementi la scintilla, la scossa, la decomposizione elettro-chimica ec.

Ho creduto una volta che la cagione della corrente sviluppata nelle esposte circostanze, stesse nella combinazione dell ossigene e dell' idrogene operata per mezzo dell' arqua . Oggi, rinunziando affatto a questa opinione, è per me provato all'evidenza che i due gazidrogene e ossigene agiscono separatamente e che in tanto la Pila di Grôve è una combinazione favorevole in quanto che le correnti sviluppate nel contatto di ognuno dei due gaz sul platino, hanno una direzione contraria. Tutte le analogie ci portano a credere che gli stati elettrici sviluppati dal platino a contatto di certi corpi gazosi intervengono nei fenomeni ben noti della forza catalitica di Berzelius .

Prof. Carlo Matteucci.

\title{
UNA CALDAJA A VAPORE
}

\section{CHE FA LE VECI DI UNA MACCHINA ELETTRICA}

Son già tre anni che presso Newcastle fu scoperto, per la prima volta, che un grande svolgimento d'elettricità arcompagnava l'escita del vapore generato in una caldaja sotto una forte pressione. Si trovò allora che il vapore e la caldaja avevano elettricità contrarie, ciò̀ positiva pel primo, e negativa per la seconda: si scoprì che la quantità sviluppata cresreva colla pressione del vapore. Si aggiunsero allora molte altre particolaritì del fenomeno, che esperimenti meglio fatti non hanno poi confermato. Parve necessario che la caldaja fosse isolata, che l'acqua non fosse pura, che una crosta di sale esistesse nella caldaja ec. Queste incertezze lasciarono gran dubbio sulla spiegazione del fenomeno; e pochi s'appagarono della esperienza con cui Peltier credeva d'imitare l'effetto della calda- 
ja di Newcastle. Alrune esperienze recenti di Amstrong ed altre più recenti di Faraday, hanno sparsa molta lure sopra questo soggetı. Ci limiteremo a descrivere l'esperienze di Armstrong da cui sembra provato con evidenza che lo sviluppo dell'elettricità nell' escita del vapore avviene per confricazione. Armstrong ha fissato ad una caldaja a vapore un lungo tubo

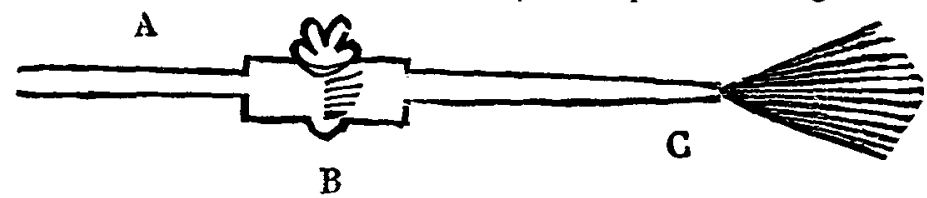

di vetro $A$, all' estremità del quale è riunito un tubo metallico portante un robinet $\mathrm{B}$ : finalmente questo robinet si termina con un sottil tubo di vetro $C$. Con questa disposizione, fattn escire il vapore, si trova che il vapore è, come al solito, elettrizzato positivamente mentre la caldaja non lo è più, ed in sua vece ha presa l' elettricita negativa il tubo metallico che porta il robinet, e che è separato dalla caldaja per mezzo del tubo di vetro. Se con un filo metallico si stabilisce una comunicazione fra la caldaja ed il robinet e si ripete l'esperienza, allora si trova di nunvo la raldaja elettrizzata. Non è dunque nella caldaja che si genera l'elettricità: ma è nel punto in rui la colonna del vapore rhe sta per escire, si trova in contatto della parete del tubo di esrita .

Faraday, in una niemoria letta alla Societa Reale di Londra, ha descritto molie esperienze dalle quali ha concluso the lo sviluppo dell' elettricità, nel getto del rapore, sotto una forte pressione, non ha luogo che allorquando il vapore si trova mescolato a goccie d'acqua. Conclude da ciò Faraday che l'elettricità è prodotta dalla confricazione dei globetti d'arqua contro le pareti del canale o contro le sostanze che si oppongono alla loro uscita, allorchè essi sono rapidamente trasporrati dalla corrente di vapore. L' effetto immediato della confricazione $\dot{e}$, in tutti $i$ casi, di rendere il rapore o l'arqua positiva, ed i solidi confricati, qualunque ne sia la loro natura, negativi. In qualche caso un filo inetalliro immerso nella corrente di vapore diviene positivo, 
28

prendendo la elettricità del vapore di cui esso non è che un semplice conduttore. I resultati di queste esperieuze possono essere grandemente modificati dalla forma dalla natura, e dalla temperatura dell'orifizio per il quale il vapore ha uscita. Il calore che impedisce la condensazione del vapore in acqua, impedisce per conseguenza lo sviluppo dell' elettricità, la quale si manifesta allorrhè si raffredda il canale sufficientemente per ottenere la condensazione del vapore. Lo sviluppo dell'elettricita dipende anche dalla qualita del fluido in movimento, relativamente alla sua facoltì condultrice. L'acqua non sviluppa l'elettricità, meno che essa non sia pura. L'aggiunta di una piccola quantiti di un sale solubile o d'acido, hasta per distruggere questa proprietì. L'aggiunta dell'olio di trementina produce uno sviluppo d'elettricitì di natura opposta a quella che fa nascer l'arqua . L'autore spiega questo fenomeno supponendo rhe ciascun globetto o particella d'acqua sia inviluppato da un leggero strato d'olio: di maniera che la confricazione ha lungo fra lo strato e i solidi contro le pareti dei quali egli passa. Un effetto simile vien prodotto dalla presenza dell'olio d'oliva: effetto ch'è di una più grande durata, stante che questolio non si dissipa tanto farilmente quanto quello di trementina. Si ot tengono dei resultati simili sostituendo una corrente d'aria alla corrente di vapore: tutte le volte che l'aria conteneva dell'umidià, i corpi solidi si elettrizavano negativamente, e la rorrente d'aria positivamente; quando poi l'aria era perfettamente serca, non vi era mai elettricità sviluppata. Il Sig. Faraday termina la sua memoria col rammentare alcune esperienze nelle quali delle polveri secche di diverse specie furonn collocate nella corrente d' aria : dice che $i$ resultati furono varj secondo la natura delle polveri impiegate e secondo altre circostanze.

Crediarro di far cosa grata ai nostri lettori riferendo qui i resultati di un'esperienza comparativa fatta dal Sig. Armstrong, onde conoscere approssimativamente la quantità d'elettricità sviliplyata dalla sua caldaja paragonandola ad una macchina 
elettrica . L'esperienza la dimostrato che la caldaja possiede una potenza eguale a sette volte quella d' una macchina eccellente che ha un disco di tre piedi di diametro, e a cui s'imprime un movimento di settanta rivoluzioni per minuto. Il confronto è stato stabilito col mezzo di un ecritatore elettro-metrico, adoperando una caldaja che consiste in un cilindro di ferro battuto, lungo 3 piedi e sei pollici, di un piede di diametro, e che riposa sopra dei sostegni di vetro.

'Tenendo le palle dell' elettro-metro alla distanza di quattro linee, si avevano 29 scariche per minuto allorchè l' eccitatore comunicava col conduttore della macchina elettrica . Questo numero era 220, allorchè lo stesso eccitatore comunicava colla caldaja isolata, e il sig. Armstrong soggiunge che di certo questo numero non è esagerato, avendosi molta pena a poter contar le scintille: tanta è la rapidità con cui si succedono. Convien peró osservare che lo sviluppo dell'elettricità è intermittente col mezzo della caldaja, giacchè di tanto in tanto convien richiuder la valvula perchè il vapore riprenda la tensione perduta.

La pressione sotto la quale il vapore deve escire, perchè l'esperienza riesra, è di 70 libbre per ogni pollice quadrato. Se si cousidera che una caldaja di un effetto eguale a quello di una macchina locomotiva può fornire molte centinaja di getti di vapore simili a quelli su cui $M$. Armstrong ha operato, potrà ognuno rappresentarsi in qualche maniera la immensa quantità di fluido eletirico che può prodursi dal vapore.

Prof. Carlo Matteucci. 